\title{
History of Recurrent Implantation Failure is Associated With the Incidence of Adverse Perinatal Outcomes in Singleton Live Births Following Frozen-Thawed Embryo Transfer Cycles
}

\author{
$\mathrm{Na} L i^{1}$, Yichun Guan ${ }^{1}$, Junjie $L_{i u^{2}}$, Bingnan Ren ${ }^{1}$, Yulin $D u^{1}$, Kexin Wang ${ }^{1}$, \\ Yongjie Zhang ${ }^{1}$ and Hua Lou ${ }^{1 *}$ \\ ${ }^{1}$ Reproductive Center, The Third Affiliated Hospital of Zhengzhou University, Zhengzhou, China, ${ }^{2}$ Henan Human Sperm \\ Bank, The Third Affiliated Hospital of Zhengzhou University, Zhengzhou, China
}

OPEN ACCESS

Edited by:

Richard Ivell,

University of Nottingham,

United Kingdom

Reviewed by:

Mariano Mascarenhas,

TFP GCRM, United Kingdom

Luca De Toni,

University of Padua, Italy

${ }^{*}$ Correspondence:

Hua Lou

1926927933@qq.com

Specialty section: This article was submitted to

Reproduction,

a section of the journal

Frontiers in Endocrinology

Received: 12 September 2021 Accepted: 14 December 2021

Published: 08 February 2022

Citation:

Li N, Guan Y, Liu J, Ren B,

Du $Y$, Wang $K$, Zhang $Y$ and Lou H (2022) History of Recurrent Implantation Failure is Associated

With the Incidence of Adverse

Perinatal Outcomes in Singleton Live Births Following Frozen-Thawed Embryo Transfer Cycles.

Front. Endocrinol. 12:774646. doi: 10.3389/fendo.2021.774646
Objective: To investigate whether patients with a history of recurrent implantation failure (RIF) are associated with adverse perinatal outcomes in singleton live births following frozen-thawed embryo transfer (FET) cycles.

Design: Retrospective cohort study.

Methods: This study analyzed the obstetric and neonatal outcomes of patients with and without a history of RIF who underwent FET cycles in a single reproductive center between January 2017 and October 2020. A total of 1,100 women with singleton live births beyond 28 weeks of gestation were included. The primary outcome measures were perinatal outcomes, especially gestational age, birthweight, preterm birth (PTB), large for gestational age (LGA), small for gestational age (SGA), congenital malformation rates, and premature rupture of the membranes (PROM). Multiple logistic regression was used to establish relationships between RIF and adverse perinatal outcomes after adjusting for relevant baseline demographics and cycle characteristics.

Result(s): The RIF group showed a preferred transfer of two embryos and cleavage embryos compared with the control group $(P<0.05)$. Regarding perinatal outcomes in singleton deliveries, women with RIF had increased rates of LBW (adjusted odds ratio [aOR] 2.027; 95\% confidence interval [Cl], 1.025-4.009), PTB (aOR 1.785; 95\% Cl, 1.0503.036), and PROM (aOR 2.259; 95\% Cl, 1.142-4.467). The incidence of congenital malformations was similar between the two groups (4.1\% vs. $2.4 \% ; P=0.759)$. Furthermore, multiple intrauterine procedures were associated with a statistically significant increased risk of PROM in RIF patients (aOR 1.537; 95\% Cl, 1.105-2.137).

Conclusions: Women with a history of RIF were associated with an increased risk of LBW, PTB, and PROM in singleton live births after FET cycles. In addition, multiple intrauterine procedures were independent risk factors for PROM.

Keywords: recurrent implantation failure, frozen-thawed embryo transfer, premature rupture of the membranes, perinatal outcomes, intrauterine procedures 


\section{INTRODUCTION}

Assisted reproductive technology (ART) has improved substantially over the past four decades and has brought hope to thousands of infertile families (1). Nevertheless, many couples still fail to become pregnant for unexplained reasons, even after multiple attempts at embryo transfer, which undoubtedly places tremendous mental pressure and economic burden on the patients and their families. This is often described as recurrent implantation failure (RIF); so far, there is no universally accepted consensus on the definition of RIF. Coughlan et al. (2) recently proposed that "women under the age of 40 years who experienced at least three fresh or frozen-thawed embryo transfer (FET) cycles and cumulatively transferred at least four good-quality embryos without getting clinical pregnancy" were considered to be RIF.

Embryo implantation is a complex process that depends on embryo quality, endometrial receptivity, and the communication between embryos and the endometrium. Over the last two decades, the management of RIF has included preimplantation genetic testing for aneuploidy in order to select only euploid embryos for transfer, or extended culture leading to embryo transfer at the blastocyst-stage, or the practice of hysteroscopy to eliminate an abnormal uterine cavity (3). Recently, a number of procedures, namely, the administration of endometrial scraping and perfusion of granulocyte colony-stimulating factor (G-CSF) before embryo transfer have been developed to improve endometrial receptivity and decrease the incidence of implantation failure in in vitro fertilization (IVF) cycles (4-6).

Previous studies have addressed how to improve pregnancy outcomes in women with multiple previous implantation failure, but the impact of a history of RIF on perinatal outcome has rarely been studied. Increasing evidences suggest that infants born after ART treatment are associated with a higher risk of low birth weight (LBW), preterm birth (PTB), small for gestational age (SGA), and also have an increased incidence of gestational diabetes, hypertensive disorders, and placenta previa, in both singleton and multiple pregnancies as compared with spontaneous conception (7-9). It is of great importance to investigate whether women with a history of multiple in vitro fertilization-embryo transfer (IVF-ET) failure are at higher risk for adverse neonatal and obstetric outcomes once they finally achieve a successful pregnancy. In this single-center study, we aimed to explore perinatal outcomes of singleton live births in patients with RIF in comparison to those who underwent a first FET cycle, in order to provide closer surveillance during pregnancy.

\section{MATERIAL AND METHODS}

\section{Study Design and Population}

This retrospective cohort study analyzed the obstetric and neonatal follow-up of singleton pregnancies following FET cycles at the Reproductive Center of the Third Affiliated Hospital of Zhengzhou University from January 2017 to
October 2020. Eligible patients were women with a history of RIF who had previously undergone at least three embryo transfer cycles and transferred no less than four good-quality cleavage embryos or three blastocysts without obtaining a pregnancy and those who accepted a first IVF/intracytoplasmic sperm injection (ICSI) treatment. All female participants were under 40 years of age. The exclusion criteria were: sperm/oocyte donation cycles, pre-implantation genetic testing (PGT) cycles, and patients with diabetes, chronic hypertension, thrombophilia, thyroid and autoimmune abnormalities. Beyond these, patients with chromosomal abnormalities, congenital uterine malformations, endometrial polyps, submucosal uterine fibroids, endometriosis, adenomyosis or hydrosalpinx, and whose endometrial thickness on the transfer day was $<7 \mathrm{~mm}$ were also excluded. In view of the retrospective nature of our study, the collection and processing of the data of patients were approved by the Ethics Review Committee of the Third Affiliated Hospital of Zhengzhou University (protocol number 2021-052-01).

\section{IVF/ICSI Protocols}

The GnRH agonist protocol and GnRH-antagonist protocol were conventional controlled ovarian stimulation (COS) protocols. For the GnRH-agonist protocol, a standard dose of triptorelin (Diphereline, Ipsen Pharma, France) was administered in the early follicular phase or luteal phase of the previous cycle for pituitary downregulation. Downregulation $[\mathrm{LH}<5 \mathrm{IU} / \mathrm{L}$, serum estradiol $<50 \mathrm{pg} / \mathrm{ml}$ ] was confirmed after 15-30 d. Exogenous Gn (Gonal-F, Merck Serono, Switzerland) was administered at doses ranging between 112.5 and $300 \mathrm{IU} / \mathrm{d}$, generally in accordance with age, body mass index (BMI), basal FSH, size and number of follicles, and estradiol levels until the follicles reached maturity. The GnRH antagonist protocol is well described in our recent study (10). When at least $40 \%$ of follicles measured $>18 \mathrm{~mm}$, human chorionic gonadotrophin (hCG, Merck Serono, Switzerland) or GnRH agonist (Dophereline, Ipsen Pharma Biotech, France) was administered to trigger oocyte maturation. Oocyte retrieval was conducted under transvaginal ultrasound guidance $36-38 \mathrm{~h}$ after trigger. IVF or ICSI were then performed, according to the sperm quality, approximately $4 \mathrm{~h}$ after oocyte retrieval.

\section{Laboratory Protocols}

All embryos were cultured at $37^{\circ} \mathrm{C}$ in $5 \% \mathrm{O}_{2}$ and $6 \% \mathrm{CO}_{2} \mathrm{G}-1$ Plus medium. Fertilization was observed $16-18 \mathrm{~h}$ after insemination. On Day 3 of embryo culture, embryo morphological evaluation was performed according to Cummins' criteria based on the percentage of fragmentation and the size and number of blastomeres (11). Good quality cleavage embryos were assessed as at least grade II embryos. In our center, 1-2 cleavage embryos of good quality are usually selected for fresh embryo transfer or cryopreservation, while the remaining embryos are transferred to blastocyst G-2 Plus medium for subsequent culture and then cryopreserved. Blastocyst quality was evaluated on Day 5 or Day 6 according to Gardner and Schoolcraft's criteria (12) based on the degree of blastocoel expansion, and morphology of the inner cell mass 
(ICM) and trophectoderm (TE). The cleavage embryos and blastocysts were scored by the same experienced embryologist, thus eliminating intra-embryologist bias when evaluating embryos. The frozen-thawed procedure is described in detail elsewhere (13).

\section{Endometrial Preparation}

In subsequent FET cycles, patients usually received a hormone replacement therapy (HRT) cycle, a natural cycle, GnRHaHRT cycle or stimulated cycle protocols for endometrial preparation before embryo transfer. In general, natural cycles were used for women with regular menstrual cycles who had the growth of their dominant follicle monitored by transvaginal ultrasonography from the 10th day of the menstrual cycle. When the diameter of the dominant follicle reached $14 \mathrm{~mm}$, patients underwent a daily ovulation urine test for LH surge detection. Ovulation occurred spontaneously or was triggered by human chorionic gonadotropin (5,000-10,000 IU; Livzon) when the urine LH surge was detected. Oral dydrogesterone (Duphaston; Solvay Pharmaceuticals BV) $10 \mathrm{mg}$ three times daily was started on the day after ovulation for luteal phase support. In stimulated cycles, women received letrozole (2.5-5.0 $\mathrm{mg} / \mathrm{d}$ started on the $3 \mathrm{rd}-5$ th $\mathrm{d}$ of the menstrual cycle) for $5 \mathrm{~d}$, with or without human menopausal gonadotropin (75-150 IU; Lizhu, Pharmaceutical) injection according to the growth of the dominant follicle; other procedures were the same as for the natural cycle.

Meanwhile, the HRT cycle was used for women with irregular menstrual cycles who received oral estradiol valerate (ValieraVR; Laboratories Recalcine) $4-8 \mathrm{mg} / \mathrm{d}$ for $12 \mathrm{~d}$ starting from the $2 \mathrm{nd}$ or $3 \mathrm{rd} \mathrm{d}$ of menstruation and the estradiol dose could be increased to a maximum of $12 \mathrm{mg} / \mathrm{d}$ depending on the thickness of the endometrium. Oral dydrogesterone (Duphaston; Solvay Pharmaceuticals BV) $10 \mathrm{mg}$ twice daily plus progesterone sustained-release vaginal gel (Xenotong, Merck Sherano, Switzerland) $90 \mathrm{mg}$ daily was started when endometrial thickness reached $\geq 7 \mathrm{~mm}$. For the GnRHa-HRT cycles, patients received downregulation therapy (Dophereline, Ipsen Pharma Biotech, France) on the 2nd to 3rd day of the menstrual cycle and then the protocol was the same as for HRT cycles when pituitary downregulation reached a satisfactory standard. Only 1-2 frozen-thawed embryos were transferred on the 6th $\mathrm{d}$ of progesterone administration. Luteal support was continued to 7 weeks of gestation if a pregnancy occurred.

\section{Outcome Measures}

Live birth was defined as birth exhibiting any signs of life after 28 weeks of gestation. In our study, the primary neonatal parameters included gestational age, preterm birth (PTB, <37 weeks), very preterm birth (VPTB, $<32$ weeks), birth weight, low birth weight (LBW, $<2500 \mathrm{~g}$ ), very low birth weight (VLBW, $1,500 \mathrm{~g}$ ), small for gestational age (SGA, birthweight is below the 10th percentile of the average body weight at the same gestational age), large for gestational age (LGA, birthweight is above the 90th percentile of the average body weight at the same gestational age), newborn sex, and congenital malformations.
In addition, we also analyzed the obstetric complications among singleton live births, including gestational hypertensive disorders, gestational diabetes mellitus (GDM), placenta previa, and premature rupture of the membranes (PROM). Gestational hypertension was defined as at least two blood pressure measurements $\geq 140 / 90 \mathrm{mmHg}$ after 20 weeks of gestation, $6 \mathrm{~h}$ apart.

\section{Statistical Analysis}

All data were analyzed by SPSS 25.0 statistical software. First, we matched the sample by 1:3 propensity score matching (PSM) between the RIF group and control group. The one-sample Kolmogorov-Smirnov (K-S) test was used to check for normality. Normally distributed continuous variables were expressed as mean \pm standard deviation and compared between two groups by Student's $t$-test. Categorical data were presented as frequencies (percentages), and were compared using Pearson chi-square or Fisher's exact test as appropriate.

Univariable and multivariable logistic regression analyses were used to further explore the association between RIF and adverse obstetric and neonatal outcomes. The results are represented as odds ratio (OR), adjusted odds ratio (aOR) and 95\% confidence interval (CI). In the subgroup analysis, multivariate regression analysis was performed for possible related variables affecting the incidence of PROM in RIF patients. $P<0.05$ was considered statistically significant.

\section{RESULTS}

A total of 1,100 singleton deliveries from FET cycles met the inclusion criteria, with 276 (25.09\%) in the RIF group and 824 (74.91\%) in the control group. The baseline demographics and main cycle characteristics for women with singleton live deliveries are described in Table 1. There were no significant differences in maternal age, maternal BMI, cause of infertility, basal FSH, and endometrial thickness on the transfer day between the two groups $(P>0.05)$. Years of infertility $(3.32 \pm$ 2.60 vs. $3.90 \pm 2.85 ; P=0.003)$ and AMH level $(4.31 \pm 2.01$ vs. $4.73 \pm 2.95 ; P=0.028)$ were lower in the RIF group than in the control group. The proportion of secondary infertility $(66.30 \%$ vs. $50.36 \% ; P<0.001)$, transfer of two embryos $(61.96 \%$ vs. $21.37 \% ; P<0.001)$ and the number of intrauterine procedures ( $5.75 \pm 1.50$ vs. $1.57 \pm 0.92 ; P<0.001)$ were significantly higher in the RIF group compared with the control group. The RIF group showed a preferred transfer of cleavage embryos. Additionally, the FET endometrial preparation protocol was also significantly different between the groups $(P<0.05)$.

Neonatal outcomes and obstetric complications for singleton births are shown in Table 2 . The mean gestational age of delivery was lower in the RIF group than in the control group (271.64 \pm $13.72 \mathrm{~d}$ vs. $273.79 \pm 10.75 \mathrm{~d}$; $P=0.008$ ). The rates of LBW were higher among infants in the RIF group than those of infants in the control group (6.16\% vs. $3.52 \%)$. The risk of being born PTB $(10.87 \%$ vs. $6.31 \%)$ and $\operatorname{VPTB}(1.81 \%$ vs. $0.49 \%)$ were significantly increased in the RIF group compared with those 
TABLE 1 | Demographic characteristics of women with a singleton birth following FET in the RIF group and control group.

\begin{tabular}{|c|c|c|c|}
\hline Characteristic & $\begin{array}{l}\text { RIF group } \\
(n=276)\end{array}$ & $\begin{array}{l}\text { Control group } \\
\text { (n = 824) }\end{array}$ & $P$-value \\
\hline Maternal age (y) & $31.92 \pm 3.73$ & $31.46 \pm 3.63$ & 0.073 \\
\hline Maternal BMI (kg/m²) & $23.43 \pm 3.03$ & $23.75 \pm 3.26$ & 0.143 \\
\hline Years of infertility (y) & $3.32 \pm 2.60$ & $3.90 \pm 2.85$ & 0.003 \\
\hline Infertility type, n (\%) & & & $<0.001^{\mathrm{a}}$ \\
\hline Primary infertility & $93(33.70)$ & 409 (49.64) & \\
\hline Secondary infertility & $183(66.30)$ & $415(50.36)$ & \\
\hline Cause of infertility, n (\%) & & & $0.528^{\mathrm{a}}$ \\
\hline Tubal factor & $97(35.14)$ & $298(36.17)$ & \\
\hline Ovulatory factor & $46(16.67)$ & $154(18.69)$ & \\
\hline Male factor & $49(17.75)$ & $130(15.78)$ & \\
\hline Combined factor & $61(22.10)$ & $174(21.12)$ & \\
\hline Unexplained & $23(8.33)$ & $68(8.25)$ & \\
\hline Basal FSH (IU/L) & $6.78 \pm 2.69$ & $6.60 \pm 2.46$ & 0.327 \\
\hline $\mathrm{AMH}(\mathrm{ng} / \mathrm{ml})$ & $4.31 \pm 2.01$ & $4.73 \pm 2.95$ & 0.028 \\
\hline Endometrial preparation protocol, n (\%) (\%) & & & $<0.001^{\mathrm{a}}$ \\
\hline HRT cycles & $130(47.10)$ & $359(43.57)$ & \\
\hline Natural cycles & 79 (28.62) & $330(40.05)$ & \\
\hline GnRHa-HRT cycles & $41(14.86)$ & $25(3.03)$ & \\
\hline Stimulated cycles & $26(9.42)$ & $110(13.35)$ & \\
\hline Endometrial thickness on transfer day(mm) & $9.38 \pm 1.61$ & $9.48 \pm 1.68$ & 0.391 \\
\hline Number of transferred embryos, $\mathrm{n}(\%)$ & & & $<0.001^{\mathrm{a}}$ \\
\hline 1 & $105(38.04)$ & $582(70.63)$ & \\
\hline 2 & $171(61.96)$ & $242(29.37)$ & \\
\hline Type of transferred embryos, n (\%) & & & $0.020^{a}$ \\
\hline Cleavage embryos & $100(36.23)$ & $237(28.76)$ & \\
\hline Blastocyst embryos & $176(63.77)$ & $587(71.24)$ & \\
\hline Number of intrauterine procedures (n) & $5.75 \pm 1.50$ & $1.57 \pm 0.92$ & $<0.001$ \\
\hline
\end{tabular}

Values are presented as mean \pm standard deviation or $n$ (\%). RIF, recurrent implantation failure; BMI, body mass index; FSH, follicle stimulating hormone; AMH, anti-Mullerian hormone; FET, frozen-thawed embryo transfer; HRT, hormones replacement therapy.

aPearson's chi-square test.

from the control group. Similarly, the RIF group was associated with a significant incidence of PROM (6.88\% vs. $3.64 \%, P=$ $0.024)$. However, no significant differences were observed in terms of the rates of VLBW, SGA, LGA, male gender, congenital malformation, hypertensive disorders, GDM, and placenta previa. After discovering that the RIF group showed a trend toward an increased risk of adverse obstetric and neonatal outcomes, we then performed multivariate logistic regression analysis to further investigate the association between RIF and these perinatal outcomes, respectively (Table 2). After adjusting for the years of infertility, infertility type, AMH, FET endometrial preparation protocol, number of transferred

TABLE 2 | Neonatal outcomes and obstetric complications for singleton births in the RIF group versus control group.

\begin{tabular}{|c|c|c|c|c|c|c|}
\hline Outcomes & RIF (n = 276) & Control (n = 824) & OR $(95 \% \mathrm{Cl})$ & $P$ value & aOR $(95 \% \mathrm{Cl})$ & $P$ value \\
\hline Gestational age (d) & $271.64 \pm 13.72$ & $273.79 \pm 10.75$ & & 0.008 & & \\
\hline PTB & $30(10.87)$ & $52(6.31)$ & $1.811(1.130-2.902)$ & 0.014 & 1.785 (1.050-3.036) & 0.032 \\
\hline VPTB & $5(1.81)$ & $4(0.49)$ & $3.782(1.008-14.186)$ & 0.049 & $3.521(0.791-15.675)$ & 0.099 \\
\hline Birth weight (g) & $3,393.45 \pm 584.81$ & $3,421.49 \pm 503.86$ & & 0.443 & & \\
\hline LBW & $17(6.16)$ & $29(3.52)$ & 2.182 (1.193-3.990) & 0.011 & 2.027 (1.025-4.009) & 0.042 \\
\hline VLBW & $2(0.72)$ & $1(0.12)$ & 6.007 (0.543-66.508) & 0.144 & $4.015(0.290-55.609)$ & 0.300 \\
\hline SGA & $10(3.62)$ & $28(3.40)$ & 1.069 (0.512-2.229) & 0.859 & $1.190(0.521-2.717)$ & 0.680 \\
\hline LGA & $54(19.57)$ & $150(18.20)$ & $1.093(0.773-1.545)$ & 0.615 & $1.192(0.809-1.758)$ & 0.375 \\
\hline Male gender & $158(57.25)$ & $455(55.22)$ & $0.921(0.669-1.213)$ & 0.557 & $1.046(0.769-1.422)$ & 0.775 \\
\hline Congenital malformations & $4(1.45)$ & $10(1.21)$ & 1.197 (1.130-2.902) & 0.763 & $0.415(0.100-1.718)$ & 0.225 \\
\hline Hypertensive disorders & $23(8.33)$ & $43(5.22)$ & $1.651(0.976-2.793)$ & 0.062 & $1.430(0.788-2.595)$ & 0.240 \\
\hline GDM & $22(7.97)$ & $66(8.01)$ & $0.995(0.602-1.645)$ & 0.984 & 0.895 (0.508-1.579) & 0.703 \\
\hline Placenta previa & $2(0.72)$ & $10(1.21)$ & $0.594(0.129-2.728)$ & 0.503 & $0.576(0.112-2.967)$ & 0.510 \\
\hline PROM & $19(6.88)$ & $30(3.64)$ & 1.957 (1.083-3.536) & 0.026 & $2.259(1.142-4.467)$ & 0.019 \\
\hline
\end{tabular}

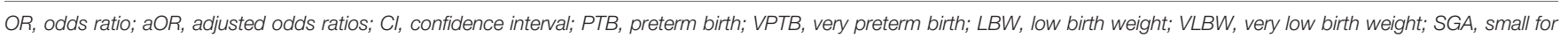

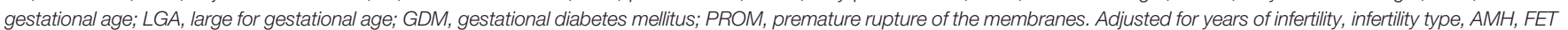
endometrial preparation protocol, number of transferred embryos, type of transferred embryos and number of intrauterine procedures. 
embryos, type of transferred embryos, and the number of intrauterine procedures, the frequencies of LBW were still higher in the RIF group compared to the control group (aOR 2.027; 95\% CI: 1.025-4.009; $P=0.042$ ). Likewise, higher risks of PTB were also observed in RIF patients (aOR 1.785; 95\% CI, $1.050-3.036 ; P=0.032$ ). Furthermore, compared with the control group, women in the RIF group were still associated with a significant increased risk of PROM (aOR 2.259; 95\% CI: 1.1424.467; $P=0.019)$.

In subgroup analysis, patients with PROM in the RIF group experienced more intrauterine procedures than those without $\operatorname{PROM}(6.53 \pm 0.96$ vs. $5.64 \pm 1.00 ; P=0.012)$. After adjusting for maternal age, maternal BMI, type of infertility, basal FSH, AMH, number of transferred embryos, type of transferred embryos, and number of intrauterine procedures, multivariable logistic analysis showed that a multiple number of intrauterine procedures was an independent risk factor for PROM in RIF patients (aOR 1.537; 95\% CI: 1.105-2.137; $P=0.014)$, as show in Table 3.

\section{DISCUSSION}

The results of this retrospective cohort analysis indicated that patients with a history of RIF are associated with adverse perinatal outcomes in singleton live births following FET cycles. We found that $6.16,10.87$, and $6.88 \%$ of RIF patients had LBW, PTB, and PROM, respectively. The risk of the above three adverse outcomes increased approximately two-fold in women with RIF compared with those from the control group. In addition, subgroup analyses showed that a multiple number of intrauterine procedures was a risk factor for PROM.

One reason for this study was that women with RIF have been shown to have an elevated risk of adverse perinatal outcomes. Earlier studies found that patients with a history of RIF had a significantly increased risk of placental abruption among singleton pregnancies (14), but our study did not find this

TABLE 3 | Adjusted odds ratios of premature rupture of the membranes by multivariate analysis of predictor variables in RIF patients.

\begin{tabular}{lcc}
\hline Predictor variable & aOR (95\% Cl) & P-value \\
\hline Maternal age & $0.987(0.857-1.136)$ & 0.853 \\
Maternal BMl & $1.104(0.951-1.281)$ & 0.193 \\
Type of infertility & 1 & \\
Primary infertility & $0.599(0.201-1.781)$ & 0.356 \\
Secondary infertility & $1.020(0.846-1.230)$ & 0.836 \\
Basal FSH & $1.229(1.005-1.504)$ & 0.051 \\
AMH & $2.044(0.557-7.498)$ & 0.281 \\
Number of transferred embryos & 1 & \\
1 & $1.680(0.459-6.153)$ & 0.433 \\
2 & $1.537(1.105-2.137)$ & 0.014 \\
Type of transferred embryos & & \\
Cleavage embryos & & \\
Blastocyst embryos & & \\
Number of intrauterine procedures & & \\
\hline
\end{tabular}

aOR, adjusted odds ratios; $\mathrm{Cl}$, confidence interval; $\mathrm{BMI}$, body mass index; $\mathrm{FSH}$, follicle stimulating hormone; $\mathrm{AMH}$, anti-Mullerian hormone. difference. To our knowledge, although the sample size of our study was limited, this is the first report to analyze the relationship between RIF and neonatal outcomes and obstetric complications in singleton live births only after FET cycles.

Our study showed singleton newborns conceived by women with a history of RIF are more likely to exhibit LBW. Preterm birth and intrauterine fetal growth restriction have long been known to be major factors affecting LBW. Premature infants are limited by the time of birth, that is, the duration of pregnancy is short, and the fetus is underdeveloped in the mother's uterus, resulting in a significantly higher incidence of LBW than fullterm infants. The research of Christine et al. and Victora et al. shows that premature delivery can significantly increase the incidence of $\operatorname{LBW}(15,16)$. In our cohort, we found that 18 of 19 LBW infants in the RIF group were caused by PTB, which further showed that PTB is an important factor leading to LBW.

Additionally, the current study also showed that RIF was associated with statistically significant increased risks of PTB and PROM. Preterm delivery reflects the growth of the fetus and is strongly associated with neonatal mortality, which may have a great impact on a child's health in the long run. It is not difficult to understand that PROM itself can cause premature delivery. Emma et al. postulated that defective implantation causes pregnancy loss, while partially impaired implantation may lead to placenta-associated complications (17). Furthermore, with the intensive study of the mechanisms involved in embryoendometrial cross-talk and embryo implantation, most patients with RIF also exhibit an increased blood flow resistance of the spiral artery, which will reduce blood supply to the placenta and lead to placental ischemia and hypoxia, finally resulting in adverse pregnancy outcomes (18-20).

To our surprise, we also noted that multiple intrauterine procedures were an independent risk factor for PROM. When it comes to treatment of RIF patients, the most commonly proposed, used, and studied interventions to overcome RIF are preconception therapies, and experimental intrauterine therapies including endometrial scratching, endometrial receptivity analysis, hysteroscopy, and intrauterine G-CSF administration (21-24). The types of therapy aim to correct disorders of endometrial receptivity, which in turn improve reproductive outcomes in RIF patients. The pathophysiologic mechanisms include an increase in the release of growth factors, interleukins, cytokines, and dendritic cell cytokines, and the induction of endometrial decidualization (25). However, results from these clinical trials are currently conflicting in women with RIF undergoing ART treatment and the procedure-associated complications have not been assessed (26). It is well-known that the intrauterine environment plays an important role in maternal and fetal development during pregnancy (27). IVF-ET procedures involve embryo catheter transfer and experimental intrauterine therapies through the cervix, which may result in vaginal-cervical dysbacteriosis, while the ascending infection of vaginal microorganisms into the uterus may trigger an inflammatory response, ultimately leading to PROM (28). We therefore hypothesize that intrauterine procedures alter the colonization of the intrauterine microbiota in RIF patients. In order to support the hypothesis, we will further investigate the 
inflammatory sequelae of intrauterine operations and the effects of inflammation on PROM.

The strength of our study is that it was performed at a single reproductive center, and we considered the risk factors associated with the incidence of PROM. Secondly, we excluded relevant variables known to affect perinatal outcomes. Lastly, we limited the analysis to only patients with a singleton live birth delivered from FET cycles. There were, however, some limitations in our study. First of all, a retrospective cohort study has inherent limitations. Secondly, our database lacked data on parental lifestyle habits, such as smoking and drinking, which have been shown to increase the risk of adverse pregnancy outcomes. Above all, the relevant biological mechanisms of intrauterine procedures on PROM need to be further explored.

\section{Conclusions}

In summary, our study demonstrated that women with a history of RIF are associated with an increased risk of LBW, PTB, and PROM in singleton live births conceived after FET cycles. Most importantly, multiple intrauterine procedures are a risk factor for PROM. Therefore, we suggest that RIF patients should minimize unnecessary intrauterine procedures. In addition, we should strengthen antenatal care and prenatal surveillance during pregnancy to reduce the risk of adverse perinatal outcomes.

\section{DATA AVAILABILITY STATEMENT}

The raw data supporting the conclusions of this article will be made available by the authors, without undue reservation.

\section{REFERENCES}

1. Margalioth EJ, Ben-Chetrit A, Gal M, Eldar-Geva T. Investigation and Treatment of Repeated Implantation Failure Following IVF-ET. Hum Reprod (Oxf Engl) (2006) 21(12):3036-43. doi: 10.1093/humrep/del305

2. Coughlan C, Ledger W, Wang Q, Liu F, Demirol A, Gurgan T, et al. Recurrent Implantation Failure: Definition and Management. Reprod BioMed Online (2014) 28(1):14-38. doi: 10.1016/j.rbmo.2013.08.011

3. Alex S, Neri L. Assessment and Treatment of Repeated Implantation Failure (RIF). J Assist Reprod Genet (2012) 29(11):1227-39. doi: 10.1007/s10815-0129861-4

4. Sar-Shalom Nahshon C, Sagi-Dain L, Wiener-Megnazi Z, Dirnfeld M. The Impact of Intentional Endometrial Injury on Reproductive Outcomes: A Systematic Review and Meta-Analysis. Hum Reprod Update (2019) 25(1):95113. doi: 10.1093/humupd/dmy034

5. Neelam P, Tarek G, Luciano GN. Endometrial Injury to Overcome Recurrent Embryo Implantation Failure: A Systematic Review and Meta-Analysis. Reprod BioMed Online (2012) 25(6):561-71. doi: 10.1016/j.rbmo.2012.08.005

6. Claudia S, Catherine K, Cordula F, Kaino H, Osama M, Manfred S, et al. Granulocyte-Colony Stimulating Factor as Treatment Option in Patients With Recurrent Miscarriage. Arch Immunol Ther Exp (2013) 61(2):159-64. doi: 10.1007/s00005-012-0212-z

7. Burton GJ, Jauniaux E. Pregnancy, Birth, and Infant Outcomes by Maternal Fertility Status: The Massachusetts Outcomes Study of Assisted Reproductive Technology. Am J Obstet Gynecol (2017) 217(3):327.e1-.e14. doi: 10.1016/ j.ajog.2017.04.025

8. Qin J, Liu X, Sheng X, Wang H, Gao S. Assisted Reproductive Technology and the Risk of Pregnancy-Related Complications and Adverse Pregnancy Outcomes in Singleton Pregnancies: A Meta-Analysis of Cohort Studies. Fertil Steril (2016) 105(1):73-85.e1-6. doi: 10.1016/j.fertnstert.2015.09.007

\section{ETHICS STATEMENT}

The studies were reviewed and approved by the Ethics Review Committee of the Third Affiliated Hospital of Zhengzhou University. Written informed consent for participation was not required for this study in accordance with the national legislation and the institutional requirements.

\section{AUTHOR CONTRIBUTIONS}

HL and YG proposed the design ideas. NL, BR, and YD acquired and analyzed the data. NL, KW, and YZ prepared all tables and figures. NL wrote the manuscript. HL and JL revised the manuscript. All authors contributed to the article and approved the submitted version.

\section{FUNDING}

This study was supported by grant 2018020198 from the Henan Medical Science and Technology Research Project, China.

\section{ACKNOWLEDGMENTS}

The authors would like to thank all of participants and the staff of Reproductive center, the Third Affiliated Hospital of Zhengzhou University, for their selfless assistance.

9. Henningsen AK, Pinborg A, Lidegaard O, Vestergaard C, Forman JL, Andersen AN. Perinatal Outcome of Singleton Siblings Born After Assisted Reproductive Technology and Spontaneous Conception: Danish National Sibling-Cohort Study. Fertil Steril (2011) 95(3):959-63. doi: 10.1016/ j.fertnstert.2010.07.1075

10. Lou H, Li N, Guan Y, Zhang Y, Hao D, Cui S. Association Between Morphologic Grading and Implantation Rate of Euploid Blastocyst. J Ovarian Res (2021) 14(1):18. doi: 10.1186/s13048-021-00770-8

11. Cummins J, Breen T, Harrison K, Shaw J, Wilson L, Hennessey J. A Formula for Scoring Human Embryo Growth Rates in In Vitro Fertilization: Its Value in Predicting Pregnancy and in Comparison With Visual Estimates of Embryo Quality. J In Vitro Fert Embryo Transf (1986) 3(5):284-95. doi: 10.1007/ BF01133388

12. Gardner DK, Lane M, Stevens J, Schlenker T, Schoolcraft WB. Blastocyst Score Affects Implantation and Pregnancy Outcome: Towards a Single Blastocyst Transfer. Fertil Steril (2000) 73(6):1155-8. doi: 10.1016/s00150282(00)00518-5

13. Lou H, Li N, Zhang X, Sun L, Wang X, Hao D, et al. Does the Sex Ratio of Singleton Births After Frozen Single Blastocyst Transfer Differ in Relation to Blastocyst Development? Reprod Biol Endocrinol (2020) 18(1):72. doi: 10.1186/s12958-020-00623-x

14. Chin TH, Hsu YC, Soong YK, Lee CL, Wang HS, Huang HY, et al. Obstetric and Perinatal Outcomes of Pregnancy in Patients With Repeated Implantation Failure. Taiwan J Obstet Gynecol (2019) 58(4):487-91. doi: 10.1016/j.tjog.2019.05.010

15. Victora C, Aquino E, do Carmo Leal M, Monteiro C, Barros F, Szwarcwald C. Maternal and Child Health in Brazil: Progress and Challenges. Lancet (London England) (2011) 377(9780):1863-76. doi: 10.1016/S0140-6736(11)60138-4

16. Odell C, Kotelchuck M, Chetty V, Fowler J, Stubblefield P, Orejuela M, et al. Maternal Hypertension as a Risk Factor for Low Birth Weight Infants: 
Comparison of Haitian and African-American Women. Matern Child Health $J$ (2006) 10(1):39-46. doi: 10.1007/s10995-005-0026-2

17. BG J, Eric J. Placental Oxidative Stress: From Miscarriage to Preeclampsia. J Soc Gynecol Investig (2004) 11(6):341-52. doi: 10.1016/j.jsgi.2004.03.003

18. Kitaya K, Matsubayashi H, Takaya Y, Nishiyama R, Yamaguchi K, Takeuchi T, et al. Live Birth Rate Following Oral Antibiotic Treatment for Chronic Endometritis in Infertile Women With Repeated Implantation Failure. Am J Reprod Immunol (2017) 78(5):1-8. doi: 10.1111/aji.12719

19. Ruiz-Alonso M, Blesa D, Díaz-Gimeno P, Gómez E, Fernández-Sánchez M, Carranza F, et al. The Endometrial Receptivity Array for Diagnosis and Personalized Embryo Transfer as a Treatment for Patients With Repeated Implantation Failure. Fertil Steril (2013) 100(3):818-24. doi: 10.1016/ j.fertnstert.2013.05.004

20. Kliman H, Frankfurter D. Clinical Approach to Recurrent Implantation Failure: Evidence-Based Evaluation of the Endometrium. Fertil Steril (2019) 111(4):618-28. doi: 10.1016/j.fertnstert.2019.02.011

21. Vitagliano A, Andrisani A, Alviggi C, Vitale S, Valenti G, Sapia F, et al. Endometrial Scratching for Infertile Women Undergoing a First Embryo Transfer: A Systematic Review and Meta-Analysis of Published and Unpublished Data From Randomized Controlled Trials. Fertil Steril (2019) 111(4):734-46.e2. doi: 10.1016/j.fertnstert.2018.12.008

22. El-Toukhy T, Campo R, Khalaf Y, Tabanelli C, Gianaroli L, Gordts S, et al. Hysteroscopy in Recurrent in-Vitro Fertilisation Failure (TROPHY): A Multicentre, Randomised Controlled Trial. Lancet (2016) 387(10038):261421. doi: 10.1016/S0140-6736(16)00258-0

23. Hashimoto T, Koizumi M, Doshida M, Toya M, Sagara E, Oka N, et al. Efficacy of the Endometrial Receptivity Array for Repeated Implantation Failure in Japan: A Retrospective, Two-Centers Study. Reprod Med Biol (2017) 16(3):290-6. doi: 10.1002/rmb2.12041

24. Davari-Tanha F, Shahrokh Tehraninejad E, Ghazi M, Shahraki Z. The Role of G-CSF in Recurrent Implantation Failure: A Randomized Double Blind Placebo Control Trial. Int J Reprod BioMed (2016) 14(12):737-42.
25. Nastri C, Lensen S, Gibreel A, Raine-Fenning N, Ferriani R, Bhattacharya S, et al. Endometrial Injury in Women Undergoing Assisted Reproductive Techniques. Cochrane Database Syst Rev (2015) 3:CD009517. doi: 10.1002/ 14651858.CD009517.pub4

26. Shaulov T, Sierra S, Sylvestre C. Recurrent Implantation Failure in IVF: A Canadian Fertility and Andrology Society Clinical Practice Guideline. Reprod BioMed Online (2020) 41(5):819-33. doi: 10.1016/j.rbmo.2020.08.007

27. Chen HJ, Gur TL. Intrauterine Microbiota: Missing, or the Missing Link? Trends Neurosci (2019) 42(6):402-13. doi: 10.1016/j.tins.2019.03.008

28. Moore D, Soules M, Klein N, Fujimoto V, Agnew K, Eschenbach D. Bacteria in the Transfer Catheter Tip Influence the Live-Birth Rate After In Vitro Fertilization. Fertil Steril (2000) 74(6):1118-24. doi: 10.1016/s0015-0282(00) 01624-1

Conflict of Interest: The authors declare that the research was conducted in the absence of any commercial or financial relationships that could be construed as a potential conflict of interest.

Publisher's Note: All claims expressed in this article are solely those of the authors and do not necessarily represent those of their affiliated organizations, or those of the publisher, the editors and the reviewers. Any product that may be evaluated in this article, or claim that may be made by its manufacturer, is not guaranteed or endorsed by the publisher.

Copyright $\odot 2022 \mathrm{Li}$, Guan, Liu, Ren, Du, Wang, Zhang and Lou. This is an openaccess article distributed under the terms of the Creative Commons Attribution License (CC BY). The use, distribution or reproduction in other forums is permitted, provided the original author(s) and the copyright owner(s) are credited and that the original publication in this journal is cited, in accordance with accepted academic practice. No use, distribution or reproduction is permitted which does not comply with these terms. 